41th "Jaszowiec" International School and Conference on the Physics of Semiconductors, Krynica-Zdrój 2012

\title{
Influence of Epitaxial Strain on Magnetic Anisotropy in $(\mathrm{Ga}, \mathrm{Mn}) \mathrm{As}$
}

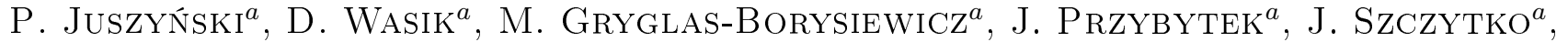 \\ A. TWARDOWSKI ${ }^{a}$ AND J. SADOWSKI ${ }^{b, c}$ \\ ${ }^{a}$ Institute of Experimental Physics, Faculty of Physics, University of Warsaw, Hoża 69, 00-681 Warsaw, Poland \\ ${ }^{b}$ Institute of Physics, PAS, al. Lotników 32/46, 02-668 Warsaw, Poland \\ ${ }^{c}$ Max-Lab, Lund University, Lund, SE-221 00, Sweden
}

Two (Ga,Mn)As samples having different magnetic anisotropy (one with in-plane easy axis and another one with out-of-plane easy axis) were studied by means of magnetotransport experiments. Anisotropy field $B_{\mathrm{A}}$ was determined for both samples as a function of temperature. For the sample having in-plane easy axis, an inversion of the direction of planar Hall effect hysteresis was observed upon increase of temperature. This result was simulated using the Stoner-Wohlfarth model.

PACS: 75.50.Pp, 75.47.-m

\section{Introduction}

( Ga,Mn) As is a diluted magnetic semiconductor, which has been considered for many years as a very promising material for possible applications in electronic devices. However, the Curie temperature achieved so far (191 K) is still too low for a real application of this material. This important problem can only be solved when a better understanding of the physical phenomena governing the magnetic properties of ( $\mathrm{Ga}, \mathrm{Mn}) \mathrm{As}$ is achieved.

Direction of the magnetization easy axis can be turned by the change of the hole concentration, temperature or strain ([1] and references therein). Our motivation was to study magnetic anisotropy of strained ( $\mathrm{Ga}, \mathrm{Mn}) \mathrm{As}$ samples with different strain as a function of temperature. Examining samples with different strain but grown in the same MBE process allows to exclude an influence of another factors.

Influence of epitaxial strain on magnetic anisotropy in two $\mathrm{Ga}_{0.94} \mathrm{Mn}_{0.06} \mathrm{As}$ layers (named sample 1 and sample 2) of $d=50 \mathrm{~nm}$ thickness was studied. Both samples were grown simultaneously in the same MBE process on [001] GaAs substrate. However, sample 2 contained $\mathrm{Ga}_{0.88} \operatorname{In}_{0.12}$ As buffer layer, which provided tensile strain. Lattice mismatch calculated basing on Vegard's law [2] was equal to $\varepsilon=-0.3 \%$ and $\varepsilon=+0.6 \%$ for sample 1 and 2 , respectively.

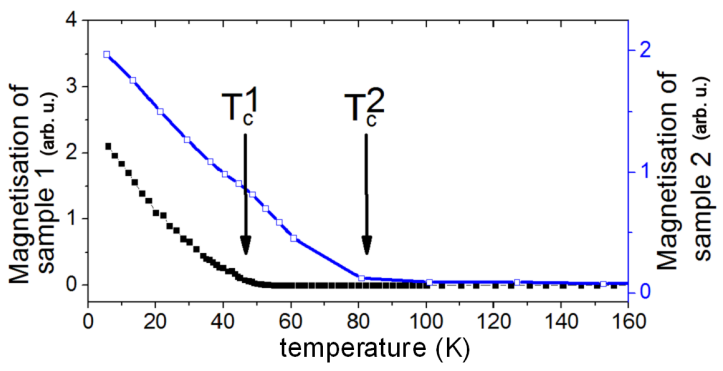

Fig. 1. Magnetization $M$ as a function of temperature. Data for sample 2 was published in [5] as sample A963.
The Curie temperature determined from magnetization measurements by means of SQUID magnetometer was $T_{\mathrm{C}}^{1} \approx 50 \mathrm{~K}$ for sample 1 and $T_{\mathrm{C}}^{2} \approx 80 \mathrm{~K}$ for sample 2 (see Fig. 1).

The Hall effect was measured as a function of temperature and magnetic field. Current flow was nearly parallel to [1-10] and [110] directions for samples 1 and 2, respectively.

\section{Anisotropy - model}

In diluted magnetic semiconductors Hall resistance $R_{X Y}$ (ratio of the Hall voltage $U_{\mathrm{H}}$ to the current $I$ ) depends on magnetization and is given by the following formula [3]:

$$
\begin{aligned}
& R_{X Y}=R_{0} B \cos \vartheta_{B}+R_{\mathrm{AHE}} M \cos \vartheta \\
& \quad+R_{\mathrm{PHE}} \sin ^{2} \vartheta \sin 2 \varphi,
\end{aligned}
$$

where $R_{0}, R_{\mathrm{AHE}}, R_{\mathrm{PHE}}$ stand for normal, anomalous and planar part of the Hall resistance, respectively (dependent on sample temperature), $\vartheta$ is an angle between magnetization $M$ and [001] axis, and $\varphi$ in an angle between $I$ and the projection of $M$ on a sample plane.

Since $R_{X Y}$ depends on magnetization direction, the Hall effect measurement is a useful tool to study magnetic anisotropy.

In the case of absence of external magnetic field $B$, magnetization $M$ is directed along an easy-axis. Application of magnetic field parallel to a hard axis forces magnetization to align parallel to $B$. The value of magnetic field $B_{\mathrm{A}}$ sufficient to align $M$ with a hard-axis is a reliable parameter of magnetic anisotropy called anisotropy field.

In the case of in-plane easy axis external magnetic field should be put perpendicularly to the sample surface (along $z$-axis). Projection of $M$ along $z$-axis is then a linear function of external magnetic field $B$ until $B_{\mathrm{A}}$ is reached

$$
M_{Z}(B)=\left\{\begin{array}{cl}
M B / B_{\mathrm{A}} & \text { for }|B| \leq B_{\mathrm{A}}, \\
M & \text { for }|B|>B_{\mathrm{A}} .
\end{array}\right.
$$

Taking into account Eqs. (1) and (2) and assuming that the planar term is negligible one can get 


$$
R_{X Y}(B)=\left\{\begin{array}{cc}
R_{\mathrm{AHE}} M B / B_{\mathrm{A}}+R_{0} B & \text { for }|B| \leq B_{\mathrm{A}}, \\
R_{\mathrm{AHE}} M+R_{0} B & \text { for }|B|>B_{\mathrm{A}}
\end{array}\right.
$$

In the case of out-of-plane anisotropy and external magnetic field parallel to the sample surface, the main term in Eq. (1) is an anomalous Hall term. $R_{X Y}$ is then proportional to the projection of $M$ along $z$-axis: $R_{X Y} \sim M_{Z}$. When $B=0, R_{X Y}$ signal achieves maximum value $\left(\vartheta_{B}=0\right)$ :

$$
R_{X Y}(B=0) \approx R_{\mathrm{AHE}} M \cos \vartheta=R_{\mathrm{AHE}} M .
$$

For strong magnetic field $B, \vartheta_{B}=90^{\circ}$ and thus $R_{X Y}=0$.

The shape of $R_{X Y}$ as a function on magnetic field can be simulated by one-domain Stoner-Wohlfarth model [4] assuming magnetostatic energy of a magnetic domain in a simple form $E=K \sin ^{2} \vartheta$, where $K$ is the anisotropy constant.

\section{Experiment and discussion}

Figure 2 presents the results of $R_{X Y}$ measurements performed for sample 2 as a function of temperature for various magnetic fields $(B \| 001)$. From this data one can easily determine $R_{X Y}$ as a function of magnetic field (inset in Fig. 2). Shape of $R_{X Y}(B)$ function shows that sample 2 exhibits an in-plane easy axis (compare with the hysteresis loops published in [5] for sample A963, which is the same sample). Anisotropy field $B_{\mathrm{A}}$ as a function of temperature was determined from experimental data using Eq. (3). We found that for temperatures close to the Curie temperature, $T \approx T_{\mathrm{c}}$, the model considered above (Eq. (3)) did not describe correctly the experimental data.

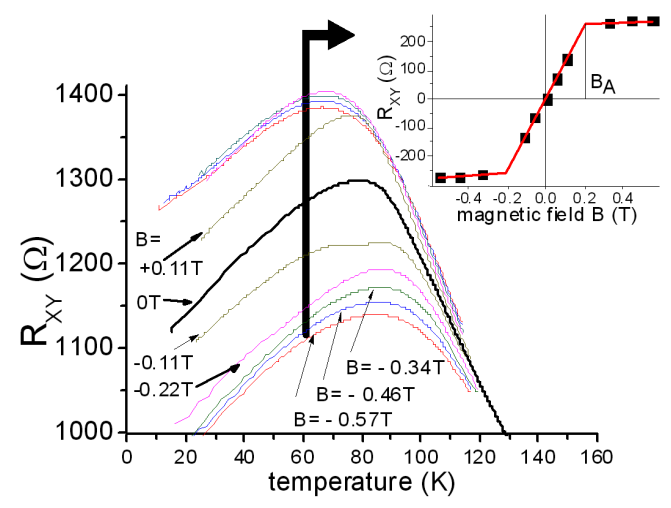

Fig. 2. Hall resistance $R_{X Y}$ measured as a function of temperature for various values of magnetic field $B$ for sample 2. For each temperature one can determine $R_{X Y}$ as a function of magnetic field (inset) and thus an anisotropy parameter $B_{\mathrm{A}}$.

Figure 3 presents the results of $R_{X Y}$ measurements performed for sample 1 as a function of magnetic field ( $B \| 110)$ for various temperatures. $R_{X Y}(B)$ hysteresis loops show that sample 1 exhibits an out-of-plane easy axis. The observed magnetization jumps are related to small field misalignment out of a sample surface. Experimental data was analyzed in the frame of the StonerWohlfarth model (anisotropy $E=K \sin ^{2} \vartheta$ ). Results of the fitting are shown in Fig. 3 (right), where solid thin line was obtained using three fitting parameters: $K$, $R_{\mathrm{AHE}} M$ and $\alpha$ ( $\alpha$ is an angle between magnetic field and sample surface). Solid thick line was calculated for the same $K$ and $R_{\mathrm{AHE}} M$ as for the thin one but that time the angle $\alpha$ was put $\alpha=0$. The above method allowed us to determine $B_{\mathrm{A}}=2 \mathrm{~K}$ for each temperature. The values of $B_{\mathrm{A}}$ obtained for both samples as a function of temperature are shown in Fig. 4.

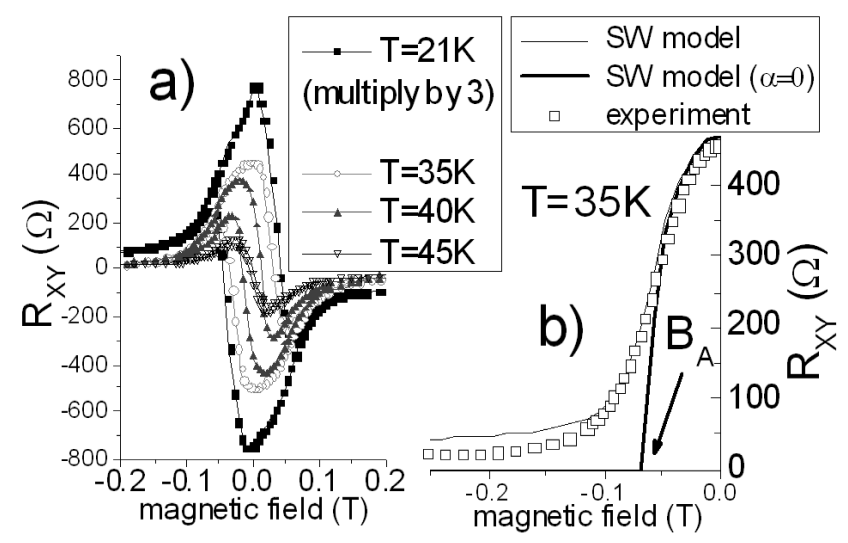

Fig. 3. (a) Hall resistance $R_{X Y}$ measured as a function of magnetic field $B$ for various temperatures for sample 1 ( $B$ in plane of the layer). (b) Data for $T=35 \mathrm{~K}$ (points) with fitted curves (see text).

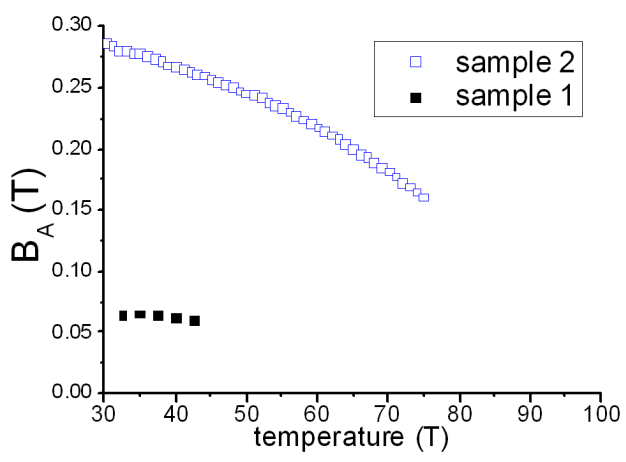

Fig. 4. Anisotropy field $B_{\mathrm{A}}$ - external magnetic field sufficient to align magnetization along hard axis determined for both samples.

One should notice that the simple procedure of determining the anisotropy field $B_{\mathrm{A}}$, considered above, is not reliable for sample 1 for $T \approx T_{\mathrm{c}}$ (amplitude of hysteresis is very small) as well as for the lowest measured temperature due to the change in the hysteresis shape. Therefore for these temperatures anisotropy field $B_{\mathrm{A}}$ was not determined. 


\section{In-plane anisotropy}

In the case of in-plane easy axis (sample 2) and in-plane external magnetic field, $B \perp[001]$ magnetization also stays in-plane, but an angle $\varphi$ between magnetization and [1-10] direction changes.

In such a case, magnetostatic energy is then given by the formula [4]:

$$
\begin{gathered}
E(\vartheta) \propto\left[\frac{B_{1}}{4} \sin ^{2} 2\left(\varphi+45^{\circ}\right)-B_{2} \sin ^{2} \varphi\right. \\
\left.-B_{3} \sin ^{2}\left(\varphi+45^{\circ}\right)-B \cos \vartheta_{B}\right],
\end{gathered}
$$

where $B_{1}$ is uniaxial and $B_{2}, B_{3}$ are biaxial anisotropy constants connected with crystalographic directions [100], [-110] and [010], respectively. $B_{1}, B_{2}, B_{3}$ depend on temperature.

Magnetization direction $(\varphi)$ minimizes Eq. (4) and thus the value of $R_{X Y}$ depends on the magnetization direction (planar term in Eq. (1)). Magnetization direction (and $R_{X Y}$ ) varies (sometimes rapidly) under influence of magnetic field. Since magnetostatic energy has more than one energetic minima, hysteresis loop in $R_{X Y}(B)$ is observed.

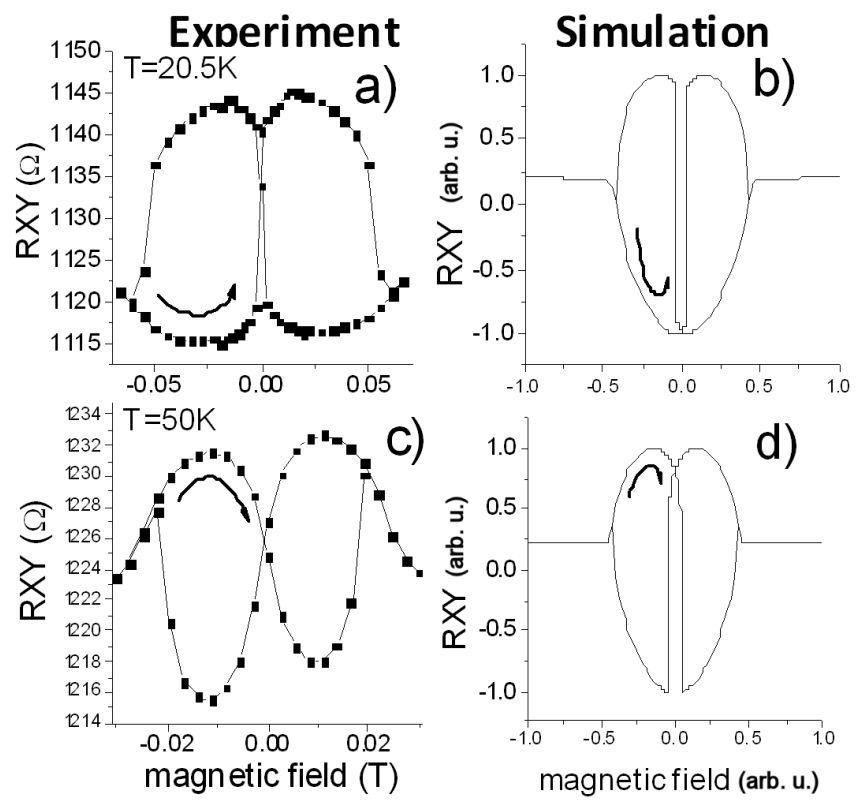

Fig. 5. Hall resistance $R_{X Y}$ measured as a function of magnetic field at various temperatures for samples 2 . Change in loop direction was modeled theoretically assuming ratio of anisotropy parameters $B_{3}: B_{2}: B_{1}$ as 125:50:0 and 125:50:1 for parts (b) and (d).

The shape of hysteresis loop depends on: $B_{1}, B_{2}, B_{3}$, $\varphi_{\mathrm{c}}$ (an angle between [110] and the current), $\varphi_{B}$ (an angle between [110] and magnetic field $B$ ). Typical hysteresis measured at $20.5 \mathrm{~K}$ and $50 \mathrm{~K}$ for sample 2 are presented in Fig. 5a,c. One should notice that the shape of hysteresis and even hysteresis direction varies with decreasing temperature. To explain this behavior numerical simulation has been performed using the Stoner-Wohlfarth model (minimizing Eq. (4)). We have found sets of parameters, which reproduce quite well turning of hysteresis direction with temperature. The angles $\varphi_{\mathrm{c}}$ and $\varphi_{B}$ cannot change during experiment. Figure 5b presents simulations for $\varphi_{\mathrm{c}}=5.9^{\circ}, \varphi_{B}=89.5^{\circ}$ and the ratio of $B_{1}: B_{2}: B_{3}$ equal to $125: 50: 0$, while Fig. 5 d presents simulations for the same values of $\varphi_{\mathrm{c}}$ and $\varphi_{B}$ but the ratio of $B_{1}: B_{2}: B_{3}$ being 125:50:1 $\left(B_{3} \neq 0\right)$. One can see that both simulated hystereses have opposite directions.

Our results show that the parameter $B_{3}$, which is sometimes assumed to be negligible [6], is important in this case. It should be noticed that even small changes of $B_{3}$ could lead to pronounced changes in the shape of hysteresis loops and one needs to care about discrete representation of the angle $\varphi$ in computational simulations and numerical artifacts.

\section{Summary}

We have studied two samples grown in the same MBE process, which revealed different magnetic anisotropy; in-plane easy axis and out-of-plane easy axis for sample with and without GaInAs buffer layer, respectively. From magnetotransport experiments, for both samples we have determined the anisotropy field $B_{\mathrm{A}}$ as a function of temperature. For the sample having in-plane easy axis we have analyzed the shape of hysteresis loops of $R_{X Y}(B)$. We have found that often neglected parameter $B_{3}$ is in this case important for reproducing our experimental data.

\section{Acknowledgments}

This paper was partially supported by National Science Centre (Poland) under grant No. DEC-2011/03/B/ ST3/03287.

\section{References}

[1] M. Sawicki, J. Magn. Magn. Mater. 300, 1 (2006).

[2] J. Sadowski, R. Mathieu, P. Svedlindh, J.Z. Domagała, J. Bak-Misiuk, K. Światek, M. Karlsteen, J. Kanski, L. Ilver, H. Åsklund, U. Södervall, Appl. Phys. Lett. 78, 3271 (2001).

[3] H. Son, S. Chung, S. Yea, S. Lee, X. Liu, J.K. Furdyna, J. Appl. Phys. 103, 07F313 (2008).

[4] K. Pappert, C. Gould, M. Sawicki, J. Wenisch, K. Brunner, G. Schmidt, L.W. Molenkamp, New J. Phys. 9, 354 (2007).

[5] M. Gryglas-Borysiewicz, A. Kwiatkowski, M. Baj, D. Wasik, J. Przybytek, J. Sadowski, Phys. Rev. B 82, 153204 (2010).

[6] D.Y. Shin, S.J. Chung, Sanghoon Lee, X. Liu, J.K. Furdyna, Phys. Rev. B 76, 035327 (2007). 